\title{
Correction to: The Relationship Among the Components of Self-compassion: A Pilot Study Using a Compassionate Writing Intervention to Enhance Self-kindness, Common Humanity, and Mindfulness
}

\author{
Aljoscha Dreisoerner ${ }^{1}$ D $\cdot$ Nina Mareen Junker ${ }^{1} \cdot$ Rolf van Dick $^{1}$
}

Published online: 8 October 2020

(c) Springer Nature B.V. 2020

\section{Correction to: Journal of Happiness Studies https://doi.org/10.1007/s10902-019-00217-4}

There was an error in computing total self-compassion, which changes one of the minor results of the paper. We reported that total self-compassion only improved significantly for the common humanity and mindfulness conditions, but not for the self-kindness condition. However, with the corrected computation, all three conditions, including the selfkindness condition, display significantly higher total self-compassion after the treatment. An updated version of Table 2 has been added to this erratum. We also corrected a minor formatting issue on Table 2.

The original article can be found online at https://doi.org/10.1007/s10902-019-00217-4.

Aljoscha Dreisoerner

dreisoerner@psych.uni-frankfurt.de

1 Institute of Psychology, Goethe University Frankfurt, Theodor-W.-Adorno-Platz 6, 60323 Frankfurt, Germany 
Table 2 Paired $t$-tests results for dependent measures among the self-kindness, common humanity, and mindfulness condition

\begin{tabular}{|c|c|c|c|c|c|}
\hline & \multicolumn{2}{|l|}{$M(S D)$} & \multirow[t]{2}{*}{$d$} & \multirow[t]{2}{*}{$t$} & \multirow[t]{2}{*}{$p$} \\
\hline & Pre & Post & & & \\
\hline \multicolumn{6}{|c|}{ Self-kindness group $(n=25)$} \\
\hline Total self-compassion & $2.95(.65)$ & $3.14(.56)$ & 0.38 & 1.91 & $.035^{*}$ \\
\hline Self-kindness & $2.91(.91)$ & $3.16(.93)$ & 0.33 & 1.63 & $.058^{+}$ \\
\hline Self-judgment & $3.09(.91)$ & $3.08(.73)$ & -0.01 & -0.06 & .478 \\
\hline Common humanity & $2.93(.81)$ & $3.06(.72)$ & 0.18 & 0.89 & .192 \\
\hline Isolation & $2.84(1.16)$ & $2.51(.83)$ & -0.34 & -1.73 & $.058^{+}$ \\
\hline Mindfulness & $3.14(.60)$ & $3.24(.65)$ & 0.18 & 0.89 & .190 \\
\hline Over-identification & $3.34(.94)$ & $3.04(.70)$ & -0.39 & -1.97 & $.045^{*}$ \\
\hline Life satisfaction & $3.50(.80)$ & $3.54(.74)$ & 0.07 & 0.34 & .737 \\
\hline WHO5 & $3.45(.73)$ & $3.50(.77)$ & 0.06 & 0.32 & .377 \\
\hline Somatic symptoms & $2.38(.65)$ & $2.29(.53)$ & -0.12 & -1.30 & .103 \\
\hline \multicolumn{6}{|c|}{ Common humanity group $(n=29)$} \\
\hline Total self-compassion & $3.11(.57)$ & $3.34(.58)$ & 0.39 & 2.11 & $.022 *$ \\
\hline Self-kindness & $3.18(.75)$ & $3.43(.77)$ & 0.30 & 1.62 & $.058^{+}$ \\
\hline Self-judgment & $2.99(.76)$ & $2.89(.76)$ & -0.13 & -0.68 & .251 \\
\hline Common humanity & $3.19(.84)$ & $3.52(.75)$ & 0.43 & 2.33 & $.014 *$ \\
\hline Isolation & $2.74(.83)$ & $2.41(.75)$ & -0.49 & -2.63 & $.028 *$ \\
\hline Mindfulness & $3.26(.87)$ & $3.34(.74)$ & 0.10 & 0.51 & .306 \\
\hline Over-identification & $3.24(.74)$ & $2.93(.79)$ & -0.46 & -2.49 & $.028 *$ \\
\hline Life satisfaction & $3.69(.59)$ & $3.92(.52)$ & 0.37 & 2.06 & $.025^{*}$ \\
\hline WHO5 & $3.48(.77)$ & $3.66(.82)$ & 0.17 & 0.87 & .196 \\
\hline Somatic symptoms & $2.48(.54)$ & $2.46(.54)$ & -0.05 & -0.22 & .414 \\
\hline \multicolumn{6}{|c|}{ Mindfulness group $(n=26)$} \\
\hline Total self-compassion & $3.06(.76)$ & $3.32(.63)$ & 0.47 & 2.40 & $.012 *$ \\
\hline Self-kindness & $2.98(1.08)$ & $3.35(.87)$ & 0.64 & 3.26 & $.014^{*}$ \\
\hline Self-judgment & $3.23(.83)$ & $2.98(.92)$ & -0.32 & -1.64 & .056 \\
\hline Common humanity & $3.29(.89)$ & $3.35(.94)$ & 0.06 & 0.31 & .378 \\
\hline Isolation & $2.84(1.10)$ & $2.48(.91)$ & -0.44 & -2.26 & $.030 *$ \\
\hline Mindfulness & $3.37(.74)$ & $3.62(.63)$ & 0.37 & 1.89 & $.035^{*}$ \\
\hline Over-identification & $3.19(.79)$ & $2.90(.68)$ & -0.45 & -2.30 & $.030 *$ \\
\hline Life satisfaction & $3.44(.95)$ & $3.53(.86)$ & 0.19 & 1.00 & .327 \\
\hline WHO5 & $3.32(1.02)$ & $3.55(1.02)$ & 0.24 & 1.22 & .118 \\
\hline Somatic symptoms & $2.37(.79)$ & $2.31(.67)$ & -0.08 & -0.64 & .267 \\
\hline
\end{tabular}

All tests one-tailed, $p$ values for the six sub-factors of self-compassion are adjusted using Benjamini and Hochberg's (1995) approach of controlling the false discovery rate in multiple testing at 5\%. Univariate ANOVAs for self-judgment, common humanity, mindfulness, WHO5, and somatic symptoms showed no significant time effects. For the sake of completeness, test statistics and unadjusted $p$ values of the post hoc tests of these variables were added in italics

${ }^{*} p<0.05 ;{ }^{+} p<0.10$

Publisher's Note Springer Nature remains neutral with regard to jurisdictional claims in published maps and institutional affiliations. 